\title{
Self inhibitors and mutagens affect fungal PCR
}

I thank the author for his reply (Luque et al., in Press). However, the presence of inhibitors and mutagens are crucial to consider when developing DNA-based diagnostic methods, which I will clarify further for the scientific community at large. A novel aspect of my interpretations is that "self" produced inhibitors are likely in the pure cultures of fungi which can be compensated for by internal amplification controls (IAC). Luque et al. (2011) did not use IAC in their (a) culture dependent PCR or (b) target foods (culture independent PCR (CIP)); hence the PCR could have been inhibited rather than the sample not containing the target DNA when they obtained a negative result.

For example, in Figs. 1 and 2 in their paper, the negative control of Penicillium aurantiogriseum may have been inhibited and hence a false negative: In Table 1 two strains of this species are positive for patulin. Similarly, the negatives in Table 1 may be false for the PCR as IAC were not employed. The PCR of the uninoculated food samples (Lane 2, Fig. 3) may have been inhibited rather than negative, and there does appear to be inhibition of the PCR on two occasions each of the apple and ham samples (i.e. four indications of inhibition). The basic problem then is a negative result from food may be false allowing the product to be considered safe when it is not - the worst possible result for a diagnostic method. An IAC remedies this problem.

The issue of "self" produced mutagens and inhibitors in cultures and food requires further explanation. As a fungus grows in culture it produces such compounds (e.g. patulin) which may affect (i.e. mutate) the DNA of the producing fungus and lead to false (a) nega- tive or (b) positive results (see references in my original letter). It is a matter of experimental design, and cultures are required to be grown to avoid these compounds, otherwise false results may occur and this was not done in Luque et al. (2011) This, and that the authors did not test all patulin producers, may lead to errors when testing food.

Finally, I did not undertake my work predominately from a taxonomic point of view as Dr Córdoba implies: It was undertaken to detect potential sources of patulin in orchards. Testing of orchard samples (including apples) using CIP further indicate this in my 2006 Food Control paper. On the other hand, fungal taxonomy is fundamental for accurate diagnostic methods to enable correct fungi to be employed and discrepancies identified.

Thank you for the opportunity to address these complex issues. Yours sincerely

Russell Paterson

R. Russell M. Paterson* IBB-Institute for Biotechnology and Bioengineering, Centre of Biological Engineering, University of Minho, Campus de Gualtar, 4710-057 Braga, Portugal

* Tel.: +351 253604 423; fax: +351253678986. E-mail address: russell.paterson@deb.uminho.pt

29 September 2011 